\title{
Introduction of the Community Rehabilitation Northern Queensland Service
}

\author{
M Mervin, R Barker, C Sealey and T Comans
}

\begin{abstract}
Objective: To analyse trends in length of hospital stay before and after the implementation of the Community Rehabilitation Northern Queensland Service (CRNQ) in Townsville, Australia.
\end{abstract}

Design: Retrospective analysis of collected administrative data provided by the data custodian Townsville Hospital Health Service District.

Setting: All patients discharged from the Townsville hospital between 1 July 2008 and 30 June 2013 for whom the Australian Refined Diagnosis Related Groups were stroke (B70), degenerative nervous system disorders (B67) or rehabilitation (Z60).

Main outcome measures: Average length of stay and total number of inpatient episodes coded stroke, degenerative nervous system disorders or rehabilitation.

Results: Length of stay for the selected diagnosis related groups was consistently ranging from 23 days to 25 days for the period 2008-2012. In the first year of full operational capacity of CRNQ (2012-13), there was an average reduction of six days in length of stay.

The major reductions in length of stay occurred in patients admitted for rehabilitation care.

Conclusions: This study adds additional evidence that earlier discharge can be facilitated for patients with neurological conditions living outside metropolitan areas when appropriate rehabilitation services are available in the community.

Abbreviations: AR-DRG - Australian Refined Diagnosis Related Groups; CRNQ - Community Rehabilitation Northern Queensland Service.

Key words: community rehabilitation; public hospital; length of stay.

\section{Merehau Mervin}

Griffith University

Menzies Health Institute Queensland

Nathan, Queensland, Australia.

\section{Ruth Barker}

James Cook University

Ringgold Standard Institution

Townsville, Queensland, Australia.

\section{Cindy Sealey}

Northern Australia Primary Health Ltd

Belgian Gardens, Queensland, Australia.

\section{Tracy Comans}

Griffith University

Menzies Health Institute Queensland

Nathan, Queensland, Australia.

\section{Correspondence:}

c.mervin@griffith.edu.au

\section{Introduction}

Rehabilitation services outside major cities in Australia are often inadequate to service the population needs. Rates of rehabilitation in hospital vary from 3.9 per 1,000 people in major cities to 2.7 per 1,000 in inner regional and 2.4 per 1,000 in outer regional areas. [1] In 2004, the median stay for rehabilitation for older people was 15 days, [2] but this is likely to be higher in regional and remote areas due to the lack of community services for people living outside major cities. Previous research has demonstrated that earlier discharge can be facilitated if appropriate community services are available. A British study found that participants could be discharged six days earlier to release capacity in the health system for the treatment of predicted increases in participants with stroke in the future. [3]

The Community Rehabilitation Northern Queensland Service (CRNQ) is an innovative community rehabilitation 
service based in Townsville, Australia. It was implemented in November 2011 to provide services to people who have experienced neurological conditions such as stroke, Parkin $\neq$ son's disease, and multiple sclerosis. It also offers services for a range of other non-specific conditions associated with functional decline. The CRNQ is a personfocused inter-professional service with student-assisted service delivery. It employs 20 full-time equivalent staff providing physiotherapy, occupational therapy, speech pathology, social work, dietetics, exercise physiology, rehabilitation nursing and rehabilitation assistant input to the programs. The CRNQ uses the International Classification of Functioning, Disability and Health (ICF) framework to guide assessment, planning, and documentation to ensure that participants have streamlined assessments and consistent goal setting and planning in place. In 2012-13, the CRNQ provided 15 different programs to 272 participants across prevention, early intervention, postdischarge and lifespan themes with a total of 10,909 occasions of service. [4]

The objective of this study was to estimate the impact of the introduction of the CRNQ service on length of hospital stay in Townsville on relevant participant groups. We analysed trends in length of hospital stay before and after the implementation of the community rehabilitation service.

\section{Methods}

We conducted a retrospective analysis of routinely collected administrative data provided by the data custodian Townsville Hospital Health Service District. Data items used in this analysis are listed in Table 1. Participants were all patients discharged from the Townsville hospital between 1 July 2008 and 30 June 2013 for whom the Australian Refined Diagnosis Related Groups (AR-DRG) were either stroke (B70), degenerative nervous system disorders (B67) or rehabilitation (Z60). Services relating to emergency departments and outpatient visits were not included. We used service related data to estimate changes in admission rates and length of stay over time. To minimise skewing of the data, patients with stays greater than 365 days were excluded from the analysis. This represented approximately $1 \%$ of all admissions over that period.

To analyse trends in length of stay, data were analysed yearly for the five years of data available. It compared four years of data before the community rehabilitation centre was implemented with the first full operational year of CRNQ (1 July 2012-31 June 2013).

\section{Findings}

Table 2 presents the diagnostic and demographic characteristics of patients admitted during 2008-2013. In 2008-09, there were 695 inpatient episodes. These increased to 958 episodes in 2011-12 and then decreased to 801 episodes in 2012-13. The average age of admitted patients remained just over 60 years and was fairly consistent over the five years. There was consistently a higher proportion of male patients ( $60 \%)$ and the majority of admitted patients received either acute or rehabilitation care over the period 2008-13.

The proportion of inpatient episodes coded stroke decreased from $43 \%$ in $2008-09$ to $29 \%$ in $2012-13$ while the proportion of inpatient episodes coded as rehabilitation increased from $43 \%$ to $55 \%$ over the five-year period. Length of stay for the selected AR-DRG grouping was consistently ranging from 23 days to 25 days for the period 2008-2012 (see Table 3). In the first year of full operational capacity of CRNQ (2012-13), there was an average reduction of six days in length of stay. The major reductions in length of stay occurred in patients

Table 1: Data Items Requested from Townsville Health Services District

\begin{tabular}{|l|l|l|}
\hline DATA & DATA ITEMS & ORIGIN \\
\hline Participant data & $\begin{array}{l}\text { Socio-economics: Age at the time of admission, gender. } \\
\text { Diagnosis: Diagnosis related group, ICD-10 diagnostic code } \\
\text { relevant to the participant's inpatient episode, diagnosis type. }\end{array}$ & $\begin{array}{l}\text { Townsville Hospital and } \\
\text { Health Service }\end{array}$ \\
$\begin{array}{l}\text { Treatment: Admission date to hospital, discharge date, transfers } \\
\text { and discharges within Townsville Hospital and Health Service, } \\
\text { referrals, and readmission, date of discharge from the episode } \\
\text { of care, type of care (e.g. acute, palliative, maintenance, } \\
\text { rehabilitation), admit source, discharge disposition, } \\
\text { length of stay. }\end{array}$ & \\
\hline
\end{tabular}


Table 2: Demographics of admitted episodes to Townsville Hospital 2008-2013

\begin{tabular}{|l|c|c|c|c|c|}
\hline CHARACTERISTIC & $2008-09$ & $2009-10$ & $2010-11$ & $2011-12$ & $2012-13$ \\
\hline Number of admitted episodes & 695 & 774 & 759 & 958 & 801 \\
Age, mean (SD) & $61.3(20.3)$ & $61.4(19.5)$ & $60.9(20.4)$ & $63.0(20.2)$ & $63.2(19.0)$ \\
Female, $\mathrm{n}(\%)$ & $294(42 \%)$ & $303(39 \%)$ & $317(42 \%)$ & $404(42 \%)$ & $323(40 \%)$ \\
\hline Care type, $\mathrm{n}(\%)$ & & & & & \\
Acute & $369(53 \%)$ & $407(53 \%)$ & $356(47 \%)$ & $385(40 \%)$ & $322(40 \%)$ \\
GEM and maintenance & $9(1 \%)$ & $10(1 \%)$ & $9(1 \%)$ & $11(1 \%)$ & $16(2 \%)$ \\
Palliative & $7(1 \%)$ & $19(2 \%)$ & $12(2 \%)$ & $39(4 \%)$ & $21(3 \%)$ \\
Rehabilitation & $310(45 \%)$ & $338(44 \%)$ & $382(50 \%)$ & $523(55 \%)$ & $442(55 \%)$ \\
\hline AR-DRG description, $\mathrm{n}(\%)$ & & & & \\
Rehabilitation & $290(43 \%)$ & $325(43 \%)$ & $373(50 \%)$ & $521(54 \%)$ & $443(55 \%)$ \\
Degenerative neurological & $95(14 \%)$ & $105(14 \%)$ & $87(12 \%)$ & $89(9 \%)$ & $123(15 \%)$ \\
Stroke & $289(43 \%)$ & $331(43 \%)$ & $290(39 \%)$ & $346(36 \%)$ & $235(29 \%)$ \\
\hline
\end{tabular}

Abbreviations: AR-DRG = Australian Refined Diagnosis Related Groups; GEM refers to Geriatric Evaluation \& Management; SD = standard deviation. ${ }^{a} A R-D R G$ codes $Z 60=$ rehabilitation includes sub-acute population with a range of conditions that are mainly neurological and musculoskeletal that led to the initial hospitalisation, $B 67=$ degenerative neurological, $B 70=$ stroke.

Table 3: Length of stay of admitted episodes to Townsville Hospital 2008-2013

\begin{tabular}{|c|c|c|c|c|}
\hline \multirow[b]{2}{*}{ DIAGNOSIS CODE AND YEAR } & \multirow[b]{2}{*}{$\begin{array}{l}\text { NUMBER OF } \\
\text { SEPARATIONS }\end{array}$} & \multicolumn{3}{|c|}{ LENGTH OF STAY (DAYS) } \\
\hline & & $\begin{array}{c}\text { MEAN } \\
\text { (STANDARD } \\
\text { DEVIATION) }\end{array}$ & $\begin{array}{c}95 \% \\
\text { CONFIDENCE } \\
\text { INTERVAL }\end{array}$ & RANGE \\
\hline \multicolumn{5}{|l|}{ ALL } \\
\hline 2008-09 & 674 & $24(39)$ & $21.2-27.1$ & $0-310$ \\
\hline 2009-10 & 761 & $23(39)$ & $20.2-25.8$ & $0-274$ \\
\hline 2010-11 & 7502 & $5(42)$ & $21.5-27.6$ & $0-335$ \\
\hline 2011-12 & 956 & $23(44)$ & $20.2-25.8$ & $0-355$ \\
\hline $2012-13$ & 801 & $17(26)$ & $15.4-19.0$ & $0-278$ \\
\hline \multicolumn{5}{|l|}{ Rehabilitation } \\
\hline 2008-09 & 290 & $43(51)$ & $37.1-49.0$ & $0-310$ \\
\hline 2009-10 & 325 & $41(52)$ & $35.4-46.7$ & $0-274$ \\
\hline $2010-11$ & 373 & $41(54)$ & $35.0-46.0$ & $0-335$ \\
\hline 2011-12 & 521 & $35(55)$ & $30.1-39.6$ & $0-355$ \\
\hline $2012-13$ & 443 & $24(31)$ & $21.0-26.9$ & $0-278$ \\
\hline \multicolumn{5}{|c|}{ Degenerative nervous system disorders } \\
\hline 2008-09 & 95 & $7(14)$ & $3.9-9.4$ & $0-65$ \\
\hline 2009-10 & 105 & $9(13)$ & $6.3-11.5$ & $0-74$ \\
\hline 2010-11 & 87 & $12(21)$ & $7.2-16.3$ & $0-135$ \\
\hline 2011-12 & 89 & $12(22)$ & $7.5-16.9$ & $0-170$ \\
\hline 2012-13 & 123 & $10(21)$ & $6.2-13.8$ & $0-206$ \\
\hline \multicolumn{5}{|l|}{ Stroke } \\
\hline 2008-09 & 289 & $11(16)$ & $9.0-12.7$ & $0-130$ \\
\hline 2009-10 & 331 & $10(19)$ & $7.8-11.8$ & $0-229$ \\
\hline 2010-11 & 290 & $8(9)$ & $6.9-8.9$ & $0-60$ \\
\hline 2011-12 & 346 & $8(11)$ & $6.8-9.1$ & $0-98$ \\
\hline $2012-13$ & 235 & $8(9)$ & $7.1-9.5$ & $0-50$ \\
\hline
\end{tabular}


Figure 1: Average length of hospital stay (days) and 95\% confidence interval of all ARDRG selected groupings

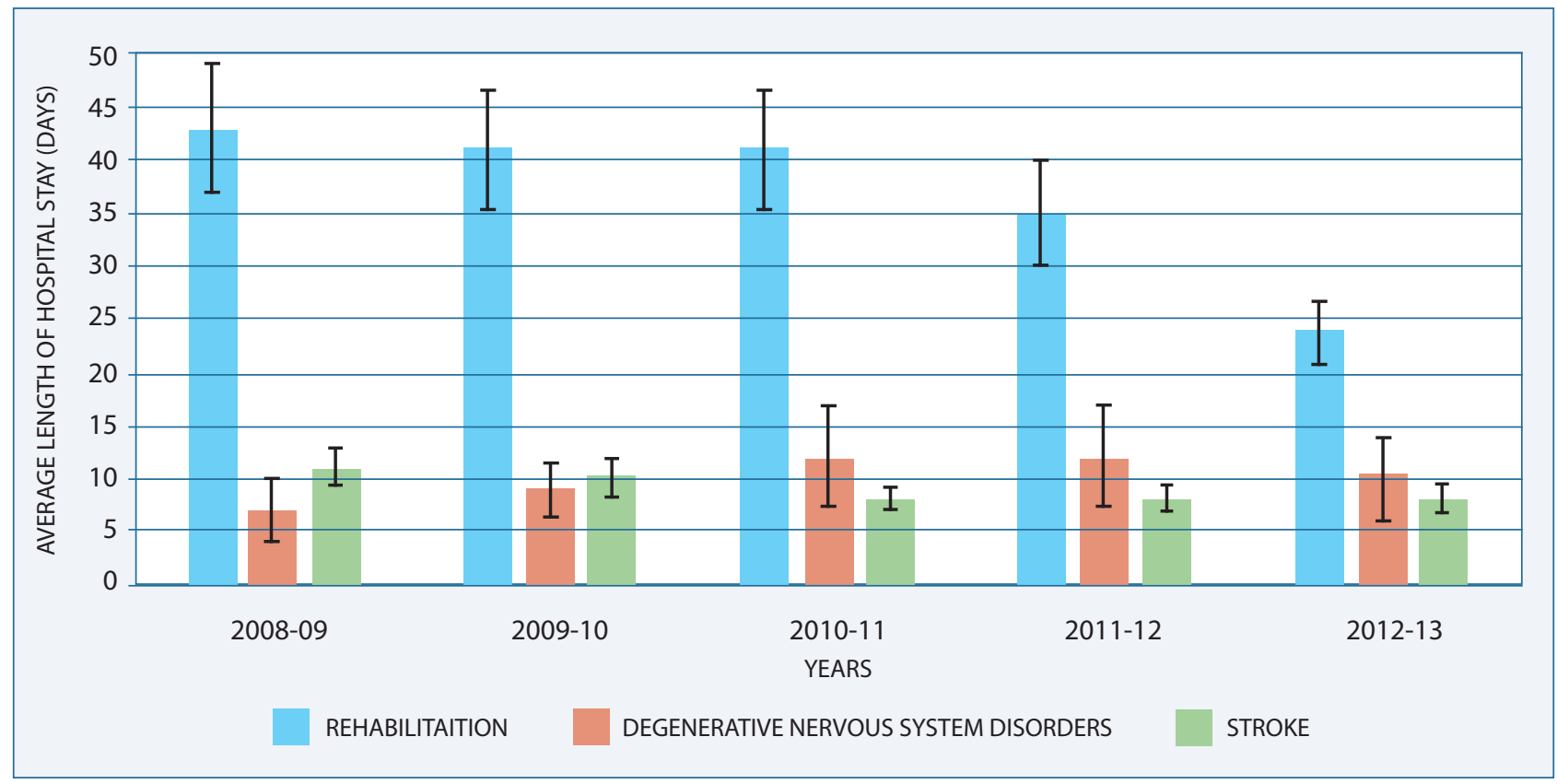

admitted for rehabilitation care (see Figure 1). The average length of stay of inpatient episodes coded rehabilitation decreased from 41 days (95\% confidence interval: 35.0-46.0) in 2010-11 to 35 days (30.1-39.6) in 2011-12 and then to 24 days (21.0-26.9) in 2012-13 (first year of full operational capacity of (RNQ).

\section{Discussion}

The study looked at the impact of the Community Rehabilitation Northern Queensland centre established in November 2011 on average length of stay at the Townsville hospital using data from Townsville Hospital Health Service District over the period 2008-13.

Our analysis showed that while the total number of inpatient episodes for whom the diagnostic group was stroke, degenerative nervous systems disorders and rehabilitation increased over the period 2008-13, the average length of hospital stay decreased by six days. The greatest change was in the average length of stay of inpatient episodes coded rehabilitation. Patients admitted for rehabilitation were younger than patients admitted for stroke and degenerative nervous system (mean age $=57$ years vs. 59 years and 70 years respectively). It is possible that patients admitted for rehabilitation were discharged to the CRNQ instead of receiving continuing care at the Townsville hospital.

Based on the 2013-14 National Efficient Pricing weights for hospitals (National efficient price of $\$ 4,993$ multiplied by cost weight of 0.18 ), the cost of inpatient rehabilitation was estimated at $\$ 909.72$ per day. [5] A six-day reduction in length of stay represents a released value to the health service. With average separations over the last five years of 788 , a total value per year of around $\$ 4.3$ million can be estimated if this were to be consistently maintained. The yearly budget for the CRNQ service (excluding the contributions for the education component of the service) was estimated at $\$ 2,792,002$, resulting in a $54 \%$ return on investment.

Our analysis only looked at three diagnosis-related groups and focused on length of hospital stay. The analysis did not consider the severity of the condition. In addition, the analysis did not examine the effectiveness of the community rehabilitation for patients with neurological conditions compared with patients who receive rehabilitation in hospital. Despite these limitations, our findings relating to shorter length of hospital stays were in concordance with other studies using similar interventions. For instance, a pilot study of rehabilitation at home found an average length of hospital stay of 14 days compared with 27 days when patients with stroke were under rehabilitation at home. [6] Our findings relating to community rehabilitation being a cost-saving alternative also concurred with other studies. [7-9]

This reduction in length of stay in rehabilitation services that generated this value amount may or may not be attributable to the operation of the CRNQ. There may have been other service initiatives operating in the hospital environment 
that have contributed to the decrease in length of stay for these patient groups. Further analysis of these data should be conducted in the next two years to evaluate whether this trend in reduction of length of stay has continued or increased.

\section{Conclusion}

Since the implementation of the Community Rehabilitation Northern Queensland centre, there has been a decrease in the average length of hospital stay of patients admitted for stroke, rehabilitation and degenerative nervous systems disorders. This study highlights the importance of access to community rehabilitation services in the management of individuals with neurological conditions living outside metropolitan areas. Clinicians may be unwilling to discharge patients who can still benefit from rehabilitation if no community services are available to continue their rehabilitation journey. Further analysis of these data should be conducted in the next two years to evaluate whether this trend in reduction of length of stay has continued or increased.

\section{Competing interests}

The authors declare that they have no competing interests.

\section{References}

1. Australian Institute of Health and Welfare. Australian hospital statistics 2009-10. Health services series no. 40. Cat. no. HSE 107. Canberra: AlHW; 2011.

2. Karmel R, Hales C, Lloyd J. Older Australians in hospital. Canberra: AlHW; 2007.

3. Beech $\mathrm{R}$, et al. Economic consequences of early inpatient discharge to communitybased rehabilitation for stroke in an inner-London teaching hospital. Stroke. 1999;30(4):29-35.

4. Townsville Mackay Medicare Local. Community Rehab NQ Annual Report 2012-13. Townsville: TMMC; 2013.

5. Independent Hospital Pricing Authority. National Efficient Price Determination 2013-14. IHPA: 2013

6. Widen Holmqvist $L$, et al. A pilot study of rehabilitation at home after stroke: a health-economic appraisal. Scandinavian Journal of Rehabilitation Medicine. 1996;28(1):9-18.

7. Anderson C, et al. Stroke rehabilitation services to accelearte hospital discharge and provide home-based care. An overview and cost analysis. PharmacoEconomics, 2002;20(8):537-552.

8. Anderson C, et al. Home or hospital for stroke rehabiltiation? Results of a randomised controlled trial. II: Cost minimisation analysis at 6 months. Stroke. 2000;31:1032-1037.

9. Brusco NK, et al. Economic evaluation of adult rehabilitation: a systematic review and meta-analysis of randomized controlled trials in a variety of settings. Arch Phys Med Rehabil. 2014;95(1): 94-116 e4. 